\title{
The Secrets of EUVE J2056-17.1
}

M. MATHIOUDAKIS, ${ }^{1}$ J. J. DRAKE ${ }^{1}$ N. CRAIG, ${ }^{1}$ D. KILKENNY, ${ }^{2}$ J. G. DOYLE, ${ }^{3}$ M. SIRK, ${ }^{1}$ J. DUPUIS, ${ }^{1}$ A. FRUSCIONE, ${ }^{1}$ C. A. CHRISTIAN, ${ }^{1}$ AND M. J. ABBOTT ${ }^{1}$

\author{
${ }^{1}$ Center for EUV Astrophysics, 2150 Kittredge Street, University of California, \\ Berkeley, CA 94720-5030, USA \\ ${ }^{2}$ South African Astronomical Observatory, PO Box 9, Observatory 7935, South Africa \\ ${ }^{3}$ Armagh Observatory, Armagh BT61 9DG, Northern Ireland
}

EUVE J2056-17.1 is one of the brightest sources in the First EUVE Source Catalog with 0.24 counts $\mathrm{s}^{-1}$ in the Deep Survey Lexan/B band. We present optical and EUV results that show this source is one of the most active late-type dwarfs. EUVE observed a large flare with energy in excess of $10^{35}$ ergs in its Lexan/B band. The quiescent optical spectrum of the source reveals strong hydrogen Balmer and $\mathrm{Ca}$ II $\mathrm{H}$ and $\mathrm{K}$ emission. A strong $\mathrm{Li} \mathrm{I} 6707 \AA$ line is also present in the spectrum. We have estimated a $\mathrm{Li}$ abundance of $\log N(\mathrm{Li})=2.5 \pm 0.4$. The high $\mathrm{Li}$ abundance and the high flare activity favors an interpretation where the enhanced $\mathrm{Li}$ is sustained by spallation reactions.

\section{Introduction}

The Extreme Ultraviolet Explorer (EUVE) is the first mission fully dedicated to extreme ultraviolet (EUV, 60-740 $\AA$ ) astronomy. The First EUVE Source Catalog (FEC) contains 410 EUV sources (Bowyer et al. 1994). The sources included in the FEC include late-type stars, white dwarfs, early-type stars, cataclysmic variables, $\mathrm{X}$-ray binaries, novae, extragalactic sources and several unidentified objects. Almost $50 \%$ of the objects detected during the all-sky survey are late-type stars, whereas in the deep survey $70 \%$ of the objects are late-type stars. This comparison shows that late-type stars are exposure time limited as compared to the remaining objects that are further away and therefore limited by absorption from the interstellar medium. These facts would suggest that a large fraction of the unidentified objects are late-type stars. The unidentified objects may constitute a potentially interesting class of objects since they are bright in the EUV and faint in optical wavelengths.

We present EUV and optical results on the source EUVE J2056-17.1, which is one of the brightest unidentified objects in the FEC. We identify the object as a new, very active flare star. An enormous flare was detected during the $E U V E$ observations. Follow up optical spectroscopy revealed strong $\mathrm{H} \alpha$ and $\mathrm{Ca}$ II H and $\mathrm{K}$ emission. The $\mathrm{Li}$ I 6707.8 $\AA$ line is also present in the spectrum.

\section{Observations and Data Reduction}

\subsection{EUV Observations}

The EUVE all-sky survey was conducted using three coaligned scanning telescopes, which point at right angles to the satellite spin axis. A fourth telescope, the Deep Survey (DS) telescope, was aligned along the spin axis and, while pointing to the anti-sun direction, advanced at $\sim 1^{\circ}$ per day along the ecliptic equator. The filter on the DS telescope comprises three sections. The center section is the Lex/B (60-200 $\AA$ ) with two panels of $\mathrm{Al} / \mathrm{C}(170-360 \AA)$ on either side. In survey mode, as the spacecraft rotated around its spin axis, a source drifted into the field of view of the DS telescope and was observed in 
the Lex/B and Al/C filters. The source makes a full revolution through the two filters during an orbital night and traces a circle passing from the Lex/B into the $\mathrm{Al} / \mathrm{C}$ filter. Useful data are collected only during orbital night, which lasts for about $1900 \mathrm{~s}$. The combination of higher effective areas and longer exposure times gave the DS Lex/B a higher sensitivity by approximately a factor of 10 compared to the all-sky survey.

EUVE J2056-17.1 was observed with the EUVE DS instrument from approximately 1992 August 2 11:00 UT to August 4 22:00 UT. The orientation of the EUVE telescopes and the geometry of the survey was such (see Haisch et al. 1993) that the three scanning telescopes observed the same position in the sky approximately three months later. Initially one of the most puzzling results was that this very bright DS source was not detected during the all-sky survey. Analysis of the DS Lex/B count rate as a function of time showed that the source gave a very large outburst during the observations.

\subsection{Optical Observations}

A search of various astronomical catalogs and databases revealed no known source at the position of EUVE J2056-17.1. An examination of the HST Guide Star Catalogue finding chart shows an object with $V \approx 10.5$ associated with the EUVE position. Groundbased spectroscopic observations of this source were obtained with the $2.1 \mathrm{~m}$ telescope at the Kitt Peak National Observatory and the $1.9 \mathrm{~m}$ telescope at the South African Astronomical Observatory. The optical spectrum of the source is characterized by strong $\mathrm{H} \alpha$ and $\mathrm{Ca}$ II H and $\mathrm{K}$ emission. The deep molecular bands of TiO (4760 $\AA), \mathrm{MgH}(4780$ $\AA)$, and $\mathrm{CaOH}$ (4780) allow us to determine a spectral type in the range of dK7e-dM0e, which would suggest that the source is at a distance of $50 \pm 12 \mathrm{pc}$.

\section{Results and Discussion}

\subsection{The EUV Flare}

The lightcurve of the flare is shown in Figure 1. The flare is characterized by a sharp rise lasting less than $4,000 \mathrm{~s}$ reaching a peak count rate of $0.42 \pm 0.02$ counts $^{-1}$. This is about 1 order of magnitude higher than the quiescent state. The main characteristics of the decay phase are an initial fast decay followed by a much longer tail. The total duration of the flare was 1.1 days. The flare was not detected in the DS Al/C. The non-detection could be partly attributed to the broad wavelength coverage of the $\mathrm{Al} / \mathrm{C}$ filter, which includes a high background contribution from the He II $304 \AA$ geocoronal emission, and partly to considerable absorption by the interstellar medium. We have determined the flare energy using the Monsignori-Fossi \& Landini (1994) line emissivities for an average coronal temperature of $10^{7} \mathrm{~K}$. EUVE spectroscopic observations of flares from active stars such as HR 1099 and AU Mic have shown that the wavelength range covered by the Lex/B band is indeed dominated by lines of Fe XVIII-Fe XXIII formed in the temperature range $10^{6.7}-10^{7.2} \mathrm{~K}$ (Brown 1994; Cully et al. 1994). The interstellar medium attenuation was taken into account using the Rumph et al. (1994) photoionization cross sections for a hydrogen column of $N_{\mathrm{HI}}=3 \times 10^{19} \mathrm{~cm}^{-2}$. We used a $\mathrm{He} \mathrm{I} / \mathrm{HI}$ ratio of 0.1 . Using these parameters, we estimate the total energy of the flare in the Lex/ $\mathrm{B}$ band to be in excess of $10^{35}$ ergs.

The flare on EUVE J2056-17.1 is one of the most energetic flares reported on a latetype dwarf. Its lightcurve is similar to the flare observed on the dMe star AU Mic (Cully et al. 1994). The AU Mic flare lasted for about 1.5 days and was characterized by an impulsive rise to the peak, followed first by a fast decay, and then by a much longer tail. The observed time scales were much longer than the radiative and cooling time scales; this led Cully et al. (1994) to conclude that the long decay phase could be explained by a 


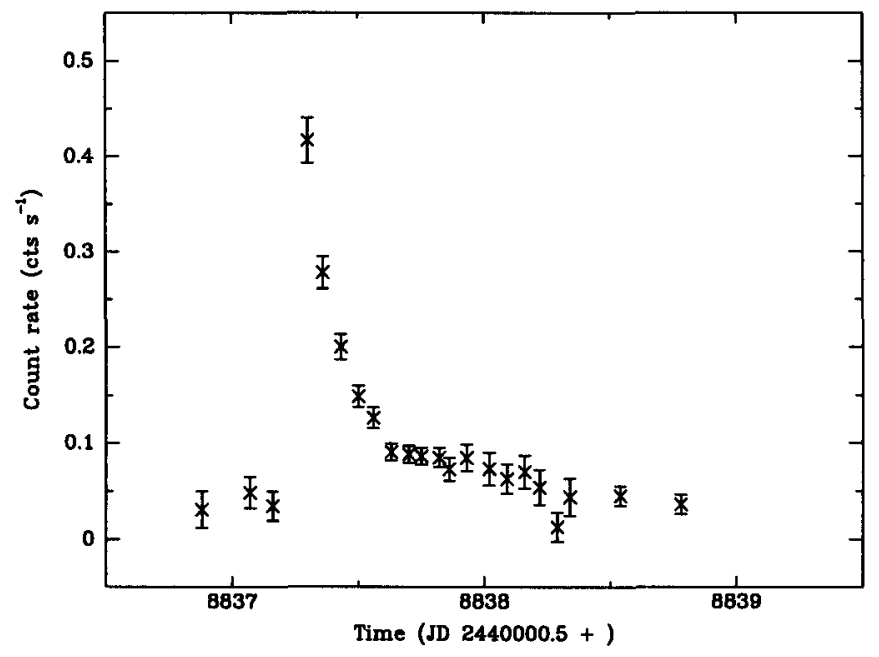

FIGURE 1. The lightcurve of the EUV flare in the DS Lex/B band (60-200 $\AA$ ). Time is in Julian Dates since JD 2440000.5. We have used one time bin per EUVE orbit for the flare. The "quiescent" data have been averaged over two orbits.

model of rapid expansion causing the plasma to become tenuous sufficiently quickly that it avoids catastrophic radiative cooling. However, in this model no additional heating is assumed during the decay phase of the event. It remains to be investigated whether the long lightcurve could be fit by a model in which the plasma cools by radiation and/or conduction with continual heating taking place throughout the duration of the event.

The EUV flares observed to date indicate that the EUV carries a large fraction of the flare's radiative losses. The high energy and long duration of this particular event being similar to the largest flares seen on RS CVn binaries (Haisch et al. 1991). The "quiescent" EUV luminosity of EUVE J2056-17.1 is $L_{\mathrm{Lex} / \mathrm{B}}=10^{30} \mathrm{ergs} \mathrm{s}^{-1}$.

\subsection{Detection of Lithium}

One of the most conspicuous lines in the optical spectrum of this source is the Li I $6707.8 \AA$ resonance line. The $\mathrm{Li}$ abundance of EUVE J2056-17.1 was estimated using the spectrum synthesis program MOOG, together with a model atmosphere generated using the MARCS program for solar metallicity (Gustafsson et al. 1975). An effective temperature of $4000 \mathrm{~K}$ and surface gravity of $\log g=4.6$ was used in the computation. These parameters were determined from the optical spectrum. The $\mathrm{Li}$ abundance derived this way is $\log N(\mathrm{Li})=2.5 \pm 0.4$. Lithium is rarely seen on late-type dwarfs with spectral types later than $\mathrm{G} 5$, and this is attributed to the fact that deep convection transports $\mathrm{Li}$ to high temperatures where it is destroyed. Could EUVE J2056-17.1 be a weak T-Tauri star? The chromospheric activity levels of EUVE J2056-17.1 are considerably lower than those of pre-main-sequence stars, they are, in fact, consistent with those of very active late-type dwarfs. The source is not associated with any clouds or star forming regions and has no entry in the IRAS Point Source Catalog. It would have been extremely interesting if EUVE J2056-17.1 turned out to be a weak T-Tauri star. However, we have no additional information at this point to support such a suggestion.

A possible interpretation that could explain the excess Li may be related to the high 


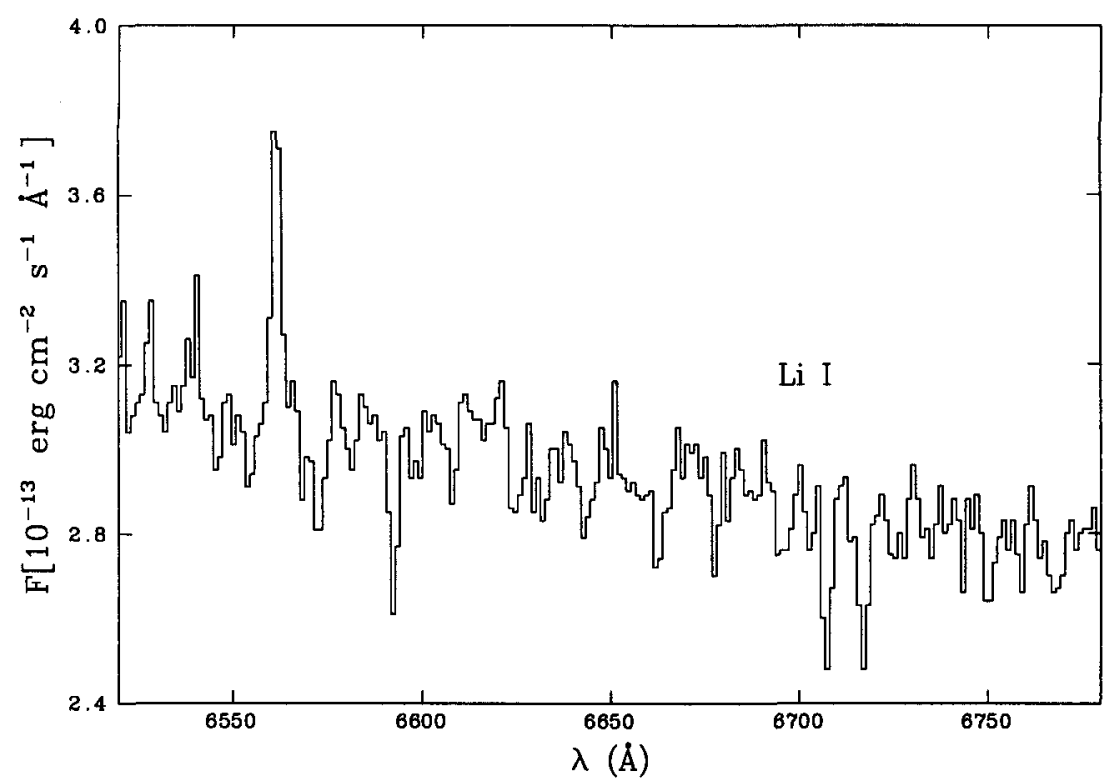

FIGURE 2. An optical spectrum of EUVE J2056-17.1 centered in the $\mathrm{H} \alpha$ region with a spectral resolution of $\sim 4 \AA$; note the presence of $\mathrm{Li}$ I $6707.8 \AA$.

flare activity of the object. Li can be produced during energetic flares by spallation reactions (Canal et al. 1980). For a $\mathrm{Li}$ abundance similar to that of EUVE J2056-17.1, Schramm et al. (1990) predict that the energy required for producing $\mathrm{Li}$ by spallation is $\approx$ $10^{46}-10^{47}$ ergs. The "quiescent" EUV luminosity of the source implies a time-averaged flare energy of $L_{\text {tot }}^{*} \approx 10^{30} \mathrm{ergs} \mathrm{s}^{-1}$. Assuming that the age of the star is similar to that of nearby flare stars, $\sim 10^{9}$ years, the energy produced is $3 \times 10^{46} \mathrm{ergs}$. If the above assumption is correct, the energy released by flares is sufficient to sustain a high $\mathrm{Li}$ abundance on this source. Both the high $\mathrm{Li}$ abundance and strong coronal emission would suggest that EUVE J2056-17.1 is a young object that recently arrived on the main sequence.

\section{Conclusions}

We have presented optical and extreme ultraviolet results showing that the previously unidentified source EUVE J2056-17.1 is a new late-type star with a spectral type in the range $\mathrm{dK} 7 \mathrm{e}-\mathrm{dM} 0 \mathrm{e}$. The "quiescent" chromospheric and coronal emission of the object show that this is one of the most active late-type dwarfs. A flare with energy in excess of $10^{35} \mathrm{ergs}$ and duration of $\sim 1.1$ days was observed in the EUVE DS Lex/B band. The optical spectrum shows a strong $\mathrm{Li}$ I $6707.8 \AA$ line. The estimated $\mathrm{Li}$ abundance is $\mathrm{log}$ $N(\mathrm{Li})=2.5 \pm 0.4$. We have discussed the possibility of Li production in the atmosphere of EUVE J2056-17.1 by spallation reactions during flares. Production of $\mathrm{Li}$ by such a 
process could delay the fast destruction occurring in the convection zone and therefore sustain an unusually high $\mathrm{Li}$ abundance.

This work has been supported by NASA Contract NAS5-30180. Research at Armagh Observatory is grant-aided by the Dept. of Education for Northern Ireland.

\section{REFERENCES}

Bowyer, S., Lieu, R., Lampton, M., Lewis, J., Wu, X., Drake, J. J., \& Malina, R. F. 1994, ApJS, 93, 569

Brown, A. 1994, in Proc. Eighth Cambridge Workshop on Cool Stars, Stellar Systems and the Sun, ed. J.P. Caillault, Astron. Soc. Pac. Conf. Ser., 64, 23

Canal, R., Isern, J., \& Sanahuja, B. 1980, ApJ, 235, 504

Cully, S. L., Fisher, G. H., AbBott, M. J., \& Siegmund, O. H. W. 1994, ApJ, 435, 449

Gustafsson, B., Bell, R. A., Eriksson, K., \& Nordlund, A. 1975, A\&A, 42, 407

Halsch, B. M., Strong, K., \& Rodono, M. 1991, ARAA, 29, 275

Haisch, B. M., BowYer, S., \& MaLINA, R. F. 1993, JBIS, 46, 331

Monsignori-Fossi, B., \& LANDinI, M. 1994, Solar Physics, 152, 81

RUMPH, T., BOWYER, S., \& VENNES, S. 1994, AJ, 107, 2108

Schramm, D. N., Steigman, G., \& Dearborn, D. S. P. 1990, ApJ, 359, L55 